\title{
CONTROL DE FORMACIONES EN VUELO DE SATÉLITES DE BAJO COSTE
}

\author{
Manuel Angulo Jerez \\ Universidad Complutense de Madrid, manuangu@ucm.es \\ Segundo Esteban San Román \\ Universidad Complutense de Madrid, segundo@dacya.ucm.es \\ José María Girón Sierra \\ Universidad Complutense de Madrid, girnosi@dacya.ucm.es \\ Óscar Rodríguez Polo \\ Universidad de Alcalá de Henares, opolo@aut.uah.es
}

\begin{abstract}
Resumen
Los satélites de bajo coste son una revolución en el sector espacial, porque permiten asumir riesgos. Un tipo de misiones arriesgadas son las formaciones en vuelo de satélites, que son muy exigentes desde el punto de vista de la algoritmia de control y del coste de propulsión. Estos satélites tienen el hándicap de su limitada capacidad de propulsión, y las prestaciones reducidas tanto de los sensores como del hardware. Para asegurar que la misión ofrezca una autonomía suficiente, es necesario estudiar modos de operación que supongan un mínimo coste de propulsión. En este artículo se presenta un análisis matemático del problema y se proponen diferentes modos de operación que minimizan el consumo del sistema de propulsión, analizando también diferentes estrategias de control.
\end{abstract}

Palabras clave: Control Orbital, Formaciones en vuelo de satélites.

\section{INTRODUCCIÓN}

Hace varias décadas, la tecnología de integración de circuitos (VLSI) dejó de ser exclusiva de las grandes compañías y comenzó a ser accesible a instituciones académicas y pequeñas compañías [1]. Esta transición fue factible gracias a la separación entre diseño y fabricación. Además, se abrió la posibilidad de compartir una oblea de silicio entre varios diseños particulares, lo que suponía una significativa reducción de costes para cada uno de los participantes. Gracias a esta idea de compartir costes, comenzó un boom de creatividad en torno al mundo del silicio que ha sido muy positivo en muchos aspectos.
Algo parecido está sucediendo actualmente en el mundo de los satélites. Lanzar un satélite suponía una gran inversión, que solo podía ser asumida por grandes compañías o centros de investigación con soporte estatal. Hoy en día es posible agrupar el lanzamiento de pequeños satélites repartiendo costes. Los picosatélites se pueden agrupar en un "Poly-PicoSatellite Orbital Deployer" (P-POD), ver figura 1, ocupando éste un hueco en el lanzador.

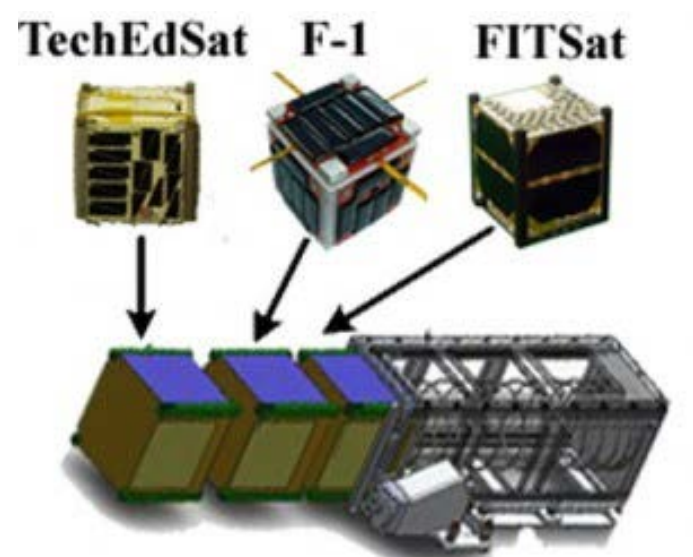

Figura 1: En un $P-P O D$ se lanzan varios satélites: FITSAT-1 (Fukuoka Institute of Technology), TechEdSat (NASA Ames) y F-1 (FPT University)

El enfoque "bajo coste" en el desarrollo de satélites no se limita al coste de lanzamiento, sino que también se aplica al hardware y al proceso de desarrollo, asumiendo riesgos de forma controlada. En estos satélites es habitual utilizar componentes COTS (Commercial Off The Shelf) y FPGAs no calificados para espacio. Debido a ello, un porcentaje significativo las misiones fallan, pero no se pueden considerar un fracaso, sino la aceptación de un riesgo que es necesario tomar en una industria aeroespacial que peca de conservadora en lo que respecta al hardware. 
Actualmente, por todo el mundo se están desarrollando misiones de bajo coste (Low Cost Satellites, LCS), con fines docentes, de investigación y comerciales. Por ejemplo, en España ya se han lanzado tres satélites de este tipo: Xacobeo (U.Vigo), Qbito (UPM) y Optos (INTA).

Este desarrollo de las misiones LCS está permitiendo a la comunidad científica plantear misiones que hasta ahora eran impensables en ámbito espacial. Un ejemplo son las formaciones en vuelo (Formation Fly, $F F$ ) de LCS. Éstas son misiones ambiciosas que ofrecen múltiples ventajas: diseño más simple, desarrollo rápido, mayor redundancia y menor coste de reemplazo. Además, las formaciones de satélites permiten alcanzar prestaciones que no son alcanzables por misiones actuales con un solo satélite.

Existen pocas experiencias satisfactorias de vuelo de satélites en FF. Una misión a destacar es PRISMA [6] cuyo objetivo era evaluar en vuelo el sistema de Guiado, Navegación y Control para futuras misiones de Rendez-vous y formaciones de vuelo. En esta misión, que no fue precisamente de bajo coste, se lanzaron unidos entre sí -y que se separaron una vez en órbita-, dos micro-satélites (Tango de $145 \mathrm{Kg}$ y Mango de 50) dotados con hardware, sensores y actuadores de muy altas prestaciones. La duración de la misión estaba limitada a tan solo 9 meses, por la limitada autonomía del sistema de corrección orbital, que utilizaba un sistema experimental de monopropulsante líquido (Green Propellant similar a la hidracina).

En [2] se analizan 39 misiones de LCS, de las cuales 12 analizan FF con pequeños satélites, siendo la mayoría misiones conceptuales. Algunas han conseguido resultados de vuelo con una autonomía muy limitada, del orden de 2 meses, por lo que tan solo sirven como demostradores tecnológicos. En este artículo se plantea economizar la acción de propulsión para aumentar la autonomía de las misiones, lo que las haría interesantes para el ámbito comercial.

En este artículo primero se analiza el problema matemático de las formaciones en vuelo de satélites. A continuación, se planifica las formaciones óptimas desde un punto de vista de coste de propulsión. Posteriormente se analizan diferentes estrategias de control. Finalmente se analizan diferentes maniobras para casos de aplicación.

\section{Control de la formación en vuelo}

\subsection{Dinámica de la formación}

A partir de las investigaciones de Hill (1878) en Lunar Theory [4], Closhessy and Wiltshire (1960) obtienen un conjunto de ecuaciones simplificadas del movimiento de la formación en vuelo [3]. Estas ecuaciones asumen muchas simplificaciones, por lo que pierden fidelidad, pero permiten analizar la esencia del movimiento relativo de los satélites de la formación.

La figura 2 muestra una formación de tres satélites. El satélite $M$ es considerado el Master de la formación y los otros dos, Si y Sj, son considerados Esclavos, con posiciones relativas al Master, $\boldsymbol{r}_{M S i}$ y $\boldsymbol{r}_{M S j}$.

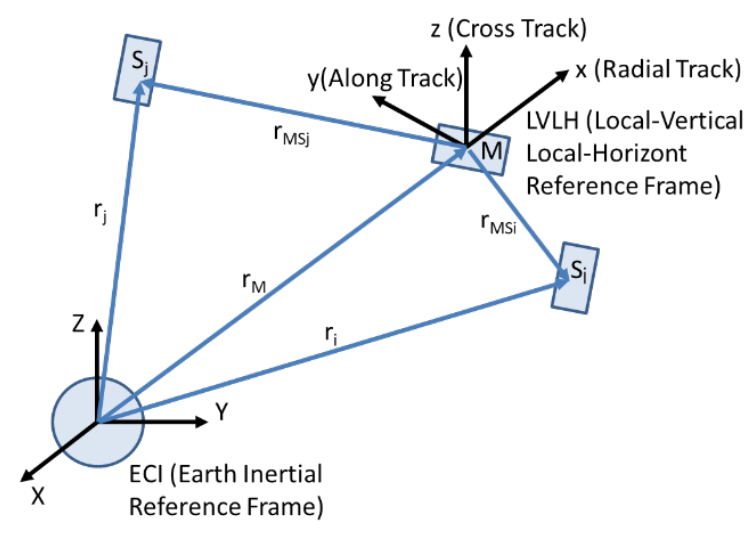

Figure 2: Sistemas de referencia utilizados para analizar la posición relativa de satélites.

El sistema de referencia inercial (Earth Centered Inertial frame, ECI) está centrado en tierra, el eje $X$ apunta al equinoccio vernal, el eje $Z$ es paralelo al momento orbital de la Tierra y el eje $Y$ es perpendicular a los anteriores. El sistema de referencia utilizado para derivar las ecuaciones de movimiento relativo es un sistema que rota centrado y en el satélite Master (Local-Vertical Local-Horizontal, LVLH). El eje $x$ apunta en la dirección radial, el eje $y$ en la dirección de la velocidad orbital y el eje $z$ en dirección normal al plano orbital.

La posición de cualquier satélite, $\boldsymbol{r}$, es modificada por la fuerza gravitatoria y otras fuerzas de menor orden, que son incluidas en el término $\boldsymbol{F}$, acorde a la ecuación diferencial (1).

$$
\ddot{\boldsymbol{r}}=-\frac{\mu \boldsymbol{r}}{r^{3}}+\boldsymbol{F}
$$

La posición relativa de un satélite esclavo respecto al satélite master vendrá gobernada por la ecuación diferencial (2).

$$
\ddot{\boldsymbol{r}}_{M S}=\frac{\mu r_{S}}{r_{S}^{3}}+\boldsymbol{F}-\frac{\mu r_{M}}{r_{M}^{3}}
$$


Operando sobre esta expresión y asumiendo que $r_{M S}^{2}$ es despreciable respecto a $r_{S}^{2}$ se puede llegar a una aproximación de primer orden para la aceleración relativa en ejes ECI, ecuación (3),

$$
\ddot{\boldsymbol{r}}_{M S} \cong-\frac{\mu}{r_{M}^{3}}\left(-3 x \widehat{\boldsymbol{I}}+\boldsymbol{r}_{M S}\right)+\boldsymbol{F}
$$

donde $\hat{\boldsymbol{I}}=\frac{r_{M}}{r_{M}}$ es el vector unitario en la dirección radial.

Para representar esta aceleración en los ejes $L V L H$ es necesario incluir los efectos inerciales de estos ejes, ecuación (4),

$$
\begin{aligned}
\ddot{\boldsymbol{r}}_{M S}^{L V L H}= & \ddot{\boldsymbol{r}}_{M S}^{E C I}-\dot{\boldsymbol{\omega}} \times \boldsymbol{r}_{M S}^{L V L H}-2 \boldsymbol{\omega} \times \dot{\boldsymbol{r}}_{M S}^{L V L H} \\
& -\boldsymbol{\omega}\left(\boldsymbol{\omega} \times \boldsymbol{r}_{M S}^{L V L H}\right)
\end{aligned}
$$

donde $\boldsymbol{\omega}$ es la velocidad angular del Sistema de referencia $L V L H$. Asumiendo una órbita circular, esta velocidad angular sería

$$
\boldsymbol{\omega}=\left[\begin{array}{l}
0 \\
0 \\
\omega
\end{array}\right] ; \text { where } \omega=\sqrt{\frac{\mu}{r_{M}^{3}}} .
$$

Utilizando la representación en $L V L H$ se llega a las ecuaciones de Closhessy-Wiltshire $(C W)(6)$,

$$
\begin{gathered}
\ddot{x}-2 \omega \dot{y}-\omega^{2} x=f_{x} \\
\ddot{y}+2 \omega \dot{x}=f_{y} \\
\ddot{z}-\omega^{2} z=f_{z}
\end{gathered}
$$

que representa la ecuación diferencial de las posiciones relativas en ejes $L V L H, \quad \boldsymbol{r}_{M S}=$ $\left[\begin{array}{lll}x & y & z\end{array}\right]^{T}$, respecto a fuerzas externas, $\boldsymbol{F}=$ $\left[\begin{array}{lll}f_{x} & f_{y} & f_{z}\end{array}\right]^{T}$, también en ejes $L V L H$.

La solución a la ecuación de $C W$ autónoma, es decir sin forzar, es (7)

$$
\begin{gathered}
x(t)=(4-3 \cos \omega t) x_{0}+\frac{\sin \omega t}{\omega} \dot{x}_{0}+\frac{2}{\omega}(1-\cos \omega t) \dot{y}_{0} \\
y(t)=6(\sin \omega t-\omega t) x_{0}+y_{0}-\frac{2}{\omega}(1-\cos \omega t) \dot{x}_{0}+\frac{4 \sin \omega t-3 \omega t}{\omega} \dot{y}_{0}
\end{gathered}
$$
posiciones y velocidades relativas iniciales en LVLH.

\subsection{Diseño de la Formación}

A partir de las ecuaciones de $C W$ se puede deducir qué formaciones son más económicas desde un punto de vista energético. La figura 3 muestra la evolución libre de una formación inicialmente radial.
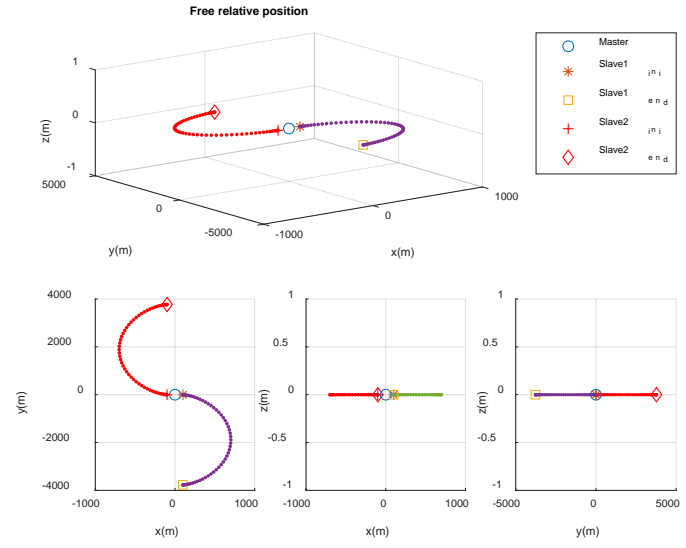

Figure 3: Deriva de una formación de dos satélites esclavos respecto al master.

Se puede apreciar que los satélites esclavos tienen deriva, cada vez se alejan más del satélite maestro. Esto se debe a que la solución a la ecuación de $C W$ tiene componentes seculares, como por ejemplo $6 \omega t x_{0}$ en $y(t)$, que aumentan continuamente con el tiempo. Compensar continuamente estas componentes seculares puede ser demasiado costoso para el sistema de propulsión, pues acortaría la autonomía de la misión. Una solución para reducir este coste de forma pasiva es elegir unas condiciones iniciales que cancelen los términos seculares.

El caso más simple es una formación along-track:

$$
\begin{aligned}
& x_{\mathbf{0}}=\mathbf{0}, \quad y_{\mathbf{0}} \neq \mathbf{0}, \quad \boldsymbol{z}_{\mathbf{0}}=\mathbf{0} \\
& \dot{x}_{0}=0 \quad \dot{y}_{0}=0 \quad \dot{z}_{0}=0
\end{aligned}
$$

En este caso, si no hubiese perturbaciones la formación se mantendría fija. Por ello el controlador de la formación tan solo tendrá que corregir los pequeños errores introducidos por las perturbaciones. Esta es la configuración ideal para recuperar energía, por lo que se puede utilizar como un modo de espera.

Otras soluciones contemplan compensar los términos entre sí. Por ejemplo, en una formación radial se pueden eliminar los términos seculares con las siguientes condiciones iniciales:

$$
\begin{aligned}
& x_{0} \neq 0, \quad y_{0}=0, \quad z_{0}=0 \\
& \dot{x}_{0}=0 \quad \dot{y}_{0}=-2 \omega x_{0} \quad \dot{z}_{0}=0
\end{aligned}
$$

Si se simulan estas condiciones iniciales, el satélite esclavo rotará en el plano orbital alrededor del satélite maestro.

Para ciertas misiones puede interesar que los satélites mantengan una distancia constante, para ello se impone la condición (8) 


$$
x^{2}+y^{2}+z^{2}=d^{2},
$$

donde la distancia de la formación viene determinada por (9)

$$
d=2 \sqrt{x_{0}^{2}+\frac{\dot{x}_{0}^{2}}{\omega}}
$$

y las otras condiciones iniciales serán:

$$
\begin{aligned}
& y_{0}=\frac{\dot{x}_{0}}{\omega}, \\
& \dot{y}_{0}=-2 \omega x_{0}, \\
& z_{0}= \pm \sqrt{3} x_{0}, \\
& \dot{z}_{0}= \pm \sqrt{3} \dot{x}_{0}
\end{aligned}
$$

De estas condiciones se deduce que las soluciones son circunferencias inclinadas respecto al horizonte. La figura 4 muestra la posición relativa del satélite esclavo respecto al satélite maestro para las condiciones iniciales anteriores.

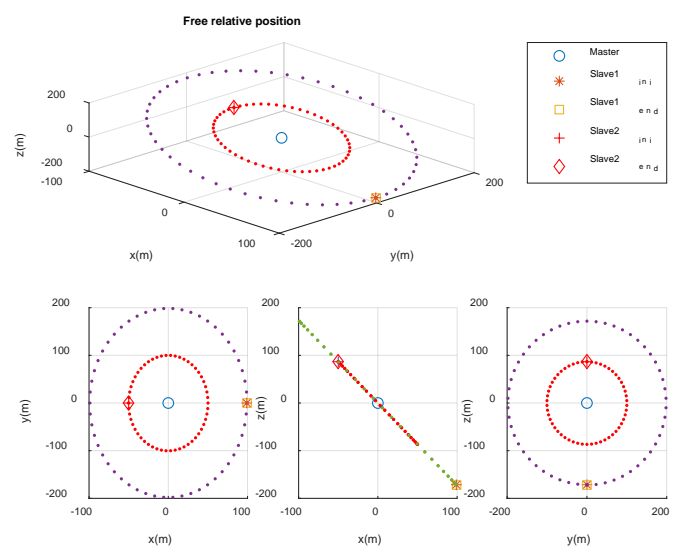

Figura 4. Resultados ideales de una formación pasiva a distancia fija.

Aunque el sistema no esté controlado, los satélites mantienen la distancia rotando alrededor del maestro. Esta sería una buena configuración para misiones con sensores a distancia fija, pues el controlador tan solo tendrá que hacer frente a perturbación y efectos no contemplados en el modelo de CW.

\subsection{Control de la Formación}

El artículo [5] hace una interesante revisión de diferentes estrategias de control de formaciones satelitales desde el punto de vista energético y de la estabilidad de controladores. En él se comparan diferentes controladores: $P D$, Lyapunov-based, Velocity Error Sliding Surface, Position Error Sliding Surface y Integrator Backstepping. El artículo llega a la conclusión que el $P D$ tiene un menor coste energético para un tiempo de estabilización similar.

Los potenciales orbitales que contemplan la ecuación de $C W$, suponen un hándicap para controlar la formación. Existe un amplio estado del arte en formaciones de robots y vehículos aéreos, terrestres o marinos, donde no existe este tipo de perturbación. Una solución sencilla para evitar los potenciales orbitales, es incluir en el control una acción directa que los anule. La ecuación $C W$ permite modelar estos potenciales, pero es necesario conocer el estado de la formación para poder calcularlos. La figura 5 muestra los diferentes bloques del lazo de control que se propone.

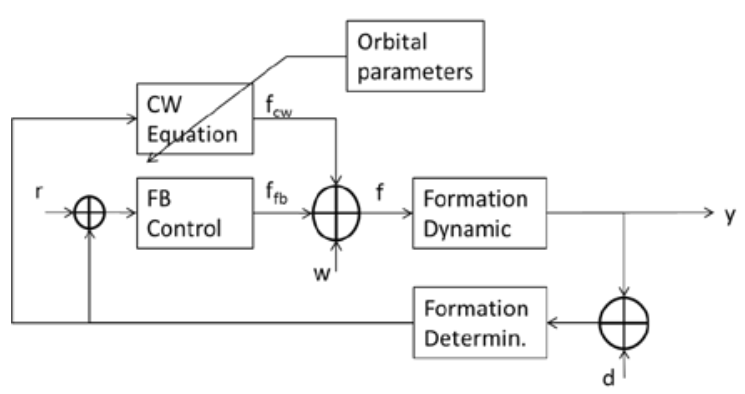

Figura 5: Esquema del sistema control necesario para anular los potenciales orbitales en formaciones de satélites.

La salida del sistema, $y$, es el estado de la formación. La referencia, $r$, es el estado deseado. La perturbación a la entrada, $w$, representa todos los efectos no modelados. La perturbación en la salida, $d$, representa los errores de medida de los sensores. El algoritmo de determinación debe estimar el estado de la formación a partir de las medidas disponibles. Los parámetros orbitales afectan a las ecuaciones de $C W$. Con la ecuación $C W$ y el estado de la formación se pueden calcular la acción $f_{C W}$ para anular los potenciales orbitales. Todos los términos no modelados en el problema pueden considerarse como una perturbación, w, en la entrada de la dinámica de la formación. Los errores que introducen estas perturbaciones deben ser atenuados mediante una realimentación del sistema, $f_{F B}$.

La configuración y dimensiones de la formación se puede traducir, utilizando $C W$, a potencia de actuación necesaria. Por ejemplo, mantener una formación along-track no requeriría apenas actuación, mientras que mantener una formación across-track requerirá una actuación $f_{C W}$ que será proporcional a la distancia que separe los satélites de la formación. Respecto a las maniobras, éstas deberán estar limitadas por la máxima capacidad de actuación y el controlador debe optimizar el coste de propulsante.

La figura 6 muestra una maniobra de paso de formación across-track a formación along-track. El satélite esclavo 1 utiliza un $P D$ como realimentación y una acción que anula los potenciales de $C W$. Se puede apreciar, que gracias a la acción de $C W$ ejecuta la maniobra mediante una trayectoria recta. El satélite 
esclavo 2 utiliza un controlador $L Q R$ basado en el modelo de $C W$ pero sin anular los potenciales de $C W$. Aparentemente realiza una trayectoria menos directa, curva y con sobre-elongación.

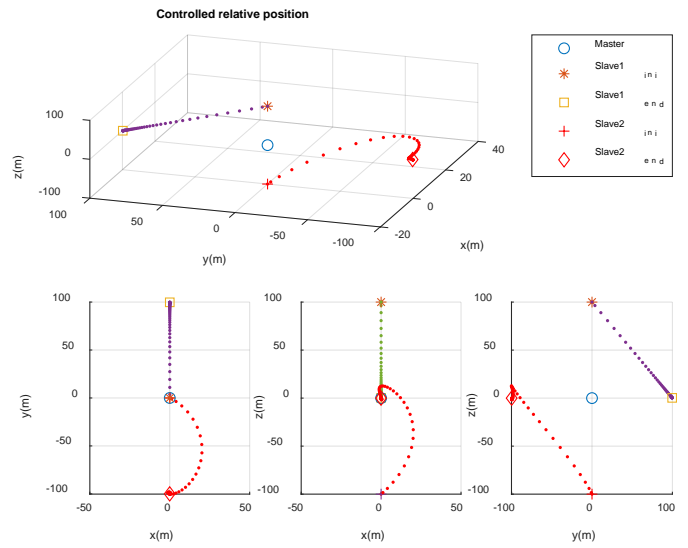

Figura 6. Simulación de una maniobra de paso de formación across-track a formación along-track.

El controlador $P D$ ha sido sintonizado para que la acción de control por cada eje no sobrepase $1 \mathrm{mN} / \mathrm{kg}$, pues es el orden de empuje que proporcionan algunos propulsores eléctricos. El controlador $L Q R$ ha sido sintonizado para obtener un tiempo de estabilización similar al obtenido con el $P D$. La figura 7 muestra información sobre el comportamiento de los controladores.
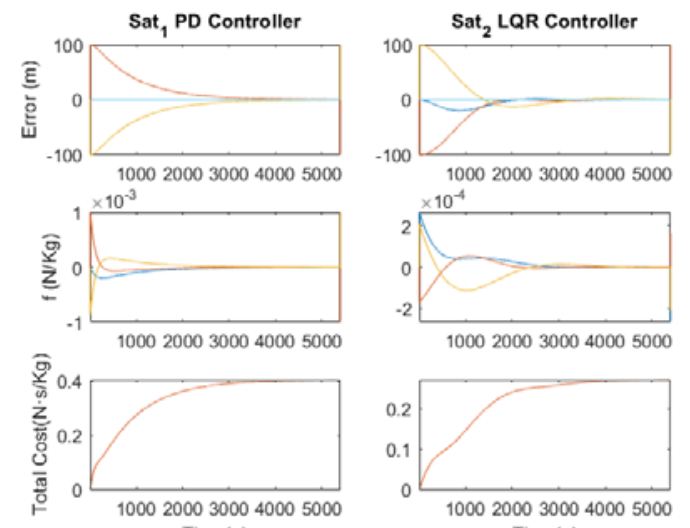

Time(s)

Figura 7. Comparativa de señales de control y coste de propulsión para los controladores $P D$ y $L Q R$.

La solución que aporta el controlador $L Q R$ tiene menor coste energético que la del controlador $P D$. La trayectoria que aparentemente era menos directa busca aprovecharse de los potenciales orbitales para alcanzar el destino con menor coste de actuación. En cambio, el controlador $P D$ directamente cancela estos potenciales, malgastando acción de control en ello. El controlador $L Q R$ es fácil de sintonizar gracias al modelo lineal de $C W$. Es necesario ampliar el estado con un integrador del error para evitar errores estacionarios.

\section{Misiones de observación de la Tierra}

La órbita típica para observar la Tierra es una órbita polar baja (Low Earth Orbit, LEO) helio congelada. En esta órbita las condiciones de iluminación a lo largo de un año completo son siempre las mismas, como muestra la figura 8.

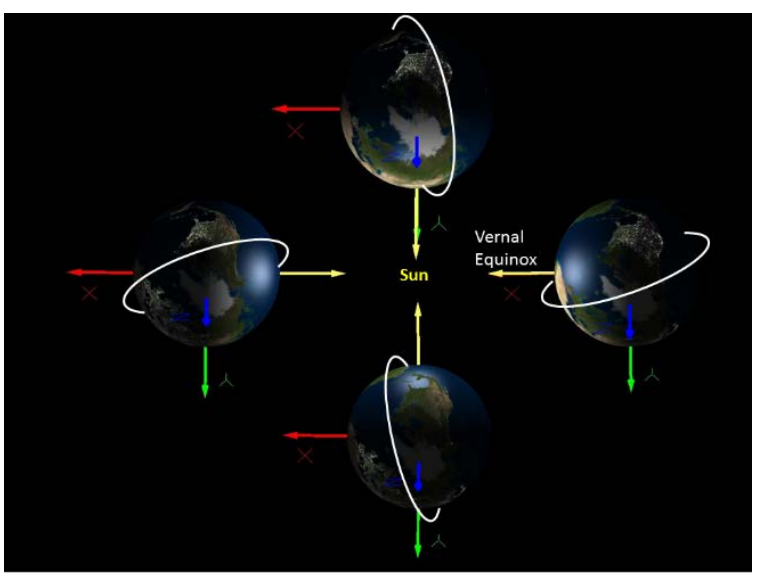

Figura 8. Evolución de una órbita Helio congelada durante un año. También muestra los ejes ECI.

Gracias a la rotación de la tierra, cualquier punto de la superficie terrestre puede ser observado cuatro veces al día, dos en iluminación y dos en eclipse. Estas órbitas tienen una altitud aproximada de $600 \mathrm{Km}$, un periodo orbital de $98 \mathrm{~min}$ (30 en eclipse), y un tiempo de pasada de unos 10 min (tiempo por encima del horizonte terrestre).

La figura 9 muestra una representación gráfica de las maniobras que se deben realizar para realizar una observación.

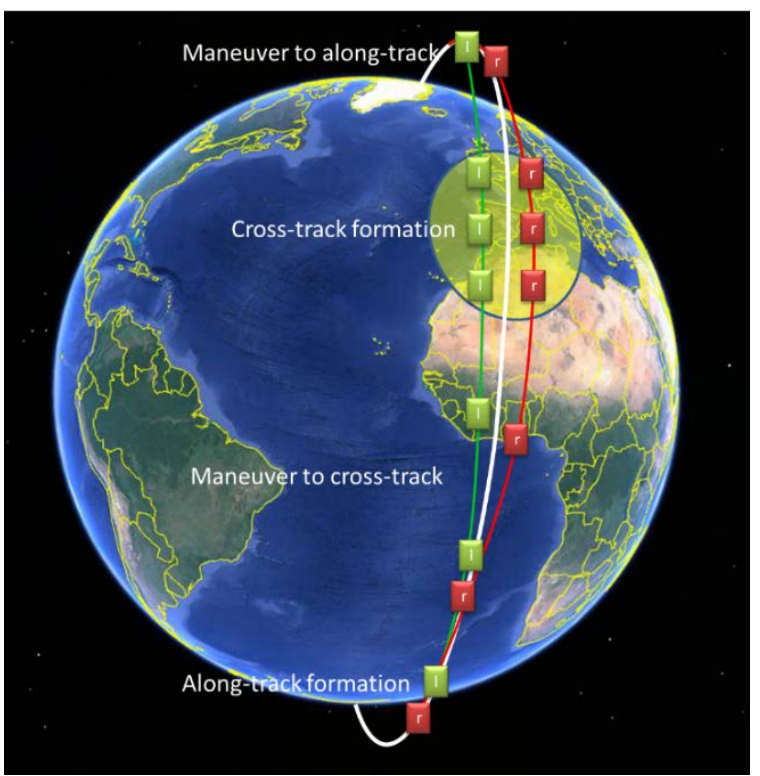

Figure 9. Representación gráfica de las maniobras para realizar una observación de la Tierra. 
Lo más conveniente es que la misión espere en una formación along-track, acumulando energía. Si en un momento determinado se requiere otra formación, p.e. across-track, se realizará una maniobra para conseguirla y mantenerla durante el tiempo requerido. Posteriormente se debe volver a la formación alongtrack.

Los controladores presentados anteriormente han sido sintonizados para economizar el coste de propulsión, por ello las maniobras necesitaban media órbita. Está claro que en este tipo de misiones tendremos un compromiso entre maniobrabilidad y autonomía. En los problemas de Rendez-vous se utiliza normalmente un control Bang-Bang, pero para mantener una formación se requiere una acción de control progresiva, no puede ser todo o nada. Un comportamiento parecido al Bang-Bang se puede obtener con un $P D$ muy agresivo que sature fácilmente la acción de control, pero manteniéndose en la zona estable. En la figura 10 se muestran una simulación de dos satélites esclavos maniobrando respecto a un satélite master virtual utilizando un control $P D$ saturado.

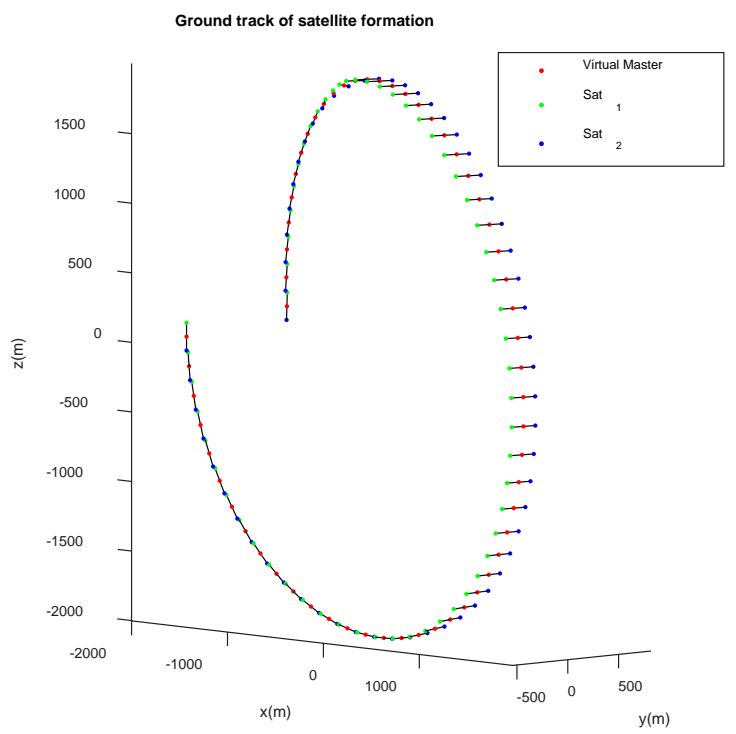

Figure 10. Traza en tierra de las maniobras para realizar una observación de la Tierra.

La figura 11 muestra las señales de control y los costes de propulsión de uno de los satélites esclavos. Se puede observar que durante las maniobras las señales de control saturan la mayoría del tiempo, pero al final actúan de forma progresiva y sin sobre-elongación. Durante la observación, $t=[3000,4000] \mathrm{s}$, es necesario compensar los potenciales gravitatorios, lo que supone que el Coste de Propulsión siga creciendo durante ese intervalo. Esto justifica que se utilice un modo de espera, p.e. along-track, en el que no haya este coste extra de propulsión.
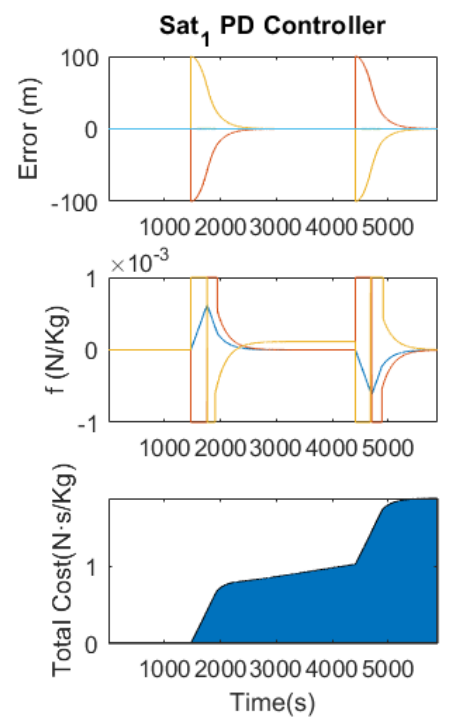

Figure 11. Representación de las maniobras a realizar para realizar una observación de la Tierra.

\section{Conclusiones}

En el artículo se ha presentado el problema del control de formaciones en vuelo de satélites. Respecto a problemas similares de formaciones de robots, en este caso existe un potencial gravitatorio que tiende a romper la formación. Se ha modelado este potencial y se han planteado un enfoque para anular su efecto mediante una acción directa.

En el caso de los satélites de bajo coste normalmente no se dispone de mucha capacidad de propulsión. Por ello es necesario afrontan estrategias de control óptimo que permitan disminuir el coste de propulsión. Se compara una estrategia de control $L Q R$ respecto al control $P D$, obteniéndose mejores resultados.

Por otro lado, hay un compromiso entre maniobrabilidad y coste de propulsión. Si se economiza mucho en propulsión trabajando en la zona lineal, las maniobras se vuelven demasiado lentas. Se ha mostrado que para acelerar las maniobras es necesario trabajar en saturación con la actuación. Por ello habrá que plantear nuevas estrategias de control óptimo que tengan en cuenta el modo de trabajo en saturación. 


\section{English summary}

\section{LOW COST SATELLITE FLYING FORMATION CONTROL}

\begin{abstract}
Low-cost satellites are a revolution in the space sector, because they allow you to take risks. Satellite flying formations are risky missions, they are very demanding from the point of view of the control algorithm and the cost of propulsion. These satellites have the handicap of their limited propulsion capacity, and the reduced performance of both sensors and hardware. To ensure that the mission offers sufficient autonomy, it is necessary to study modes of operation that suppose a minimum cost of propulsion. This article presents a mathematical analysis of the problem and proposes different modes of operation that minimize the consumption of the propulsion system, also analyzing different control strategies.
\end{abstract}

Keywords: Orbital control, satellites flying formations.

\section{Referencias}

[1] Casale-Rossi, M., (2014) "The heritage of Mead\&Conway: What has remained the same, what has changed, what was missed, and what lies ahead," Proceedings of the IEEE, vol. 102, no., 2, pp. 114-119.

[2] Bandyopadhyay, S., Subramanian, G.P., Foust, R., Morgan, D., Chung, S-J., and Hadaegh, F. (2015) "A Review of Impending Small Satellite Formation Flying Missions", 53rd AIAA Aerospace Sciences Meeting, AIAA SciTech Forum, (AIAA 2015-1623), DOI: 10.2514/6.2015-1623.

[3] Clohessy, W.H., and Wiltshire, R.S., (1960) "Terminal Guidance for Satellite Rendezvous", J. Aerospace Sciences, Vol 27, p 653.

[4] Hill, G.W., (1878) "Researches in the Lunar Theory”, American Journal of Mathematics, Vol. 1, No. 1, pp. 5-26, DOI:10.2307/2369430.

[5] Kristiansen, R., Nicklasson, P.J., (2009) "Spacecraft formation flying: A review and new results on state feedback control", Acta Astronautica, Volume 65, Issue 11, Pages 15371552, ISSN 0094-5765, DOI:10.1016/j.actaastro.2009.04.014.
[6] Persson, S., D’Amico, S., Harr, J. (2010) “Flight Results from PRISMA Formation Flying and Rendezvous Demonstration Mission,” Proceedings of the 61st IAC (International Astronautical Congress), Prague, Czech Republic, Sept. 27-Oct. 1, IAC-10-B4.2.9.

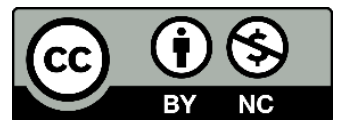

(C) 2018 by the authors. Submitted for possible open access publication under the terms and conditions of the Creative Commons Attribution CC-BY-NC $3.0 \quad$ license (https://creativecommons.org/licenses/by-nc/3.0). 